\title{
No matter how you mark the points on the fever curve - threatening shapes do not add to threat of climate change
}

\author{
Mariam Katsarava $^{1}$ (D) Helen Landmann ${ }^{1}$ (D) $\cdot$ Robert Gaschler $^{1}$ iD
}

Accepted: 18 November 2021

(c) The Author(s) 2021

\begin{abstract}
Graphs have become an increasingly important means of representing data, for instance, when communicating data on climate change. However, graph characteristics might significantly affect graph comprehension. The goal of the present work was to test whether the marking forms usually depicted on line-graphs, can have an impact on graph evaluation. As past work suggests that triangular forms might be related to threat, we compared the effect of triangular marking forms with other symbols (triangles, circles, squares, rhombi, and asterisks) on subjective assessments. Participants in Study $1(N=314)$ received 5 different line-graphs about climate change, each of them using one out of 5 marking forms. In Study 1, the threat and arousal ratings of the graphs with triangular marking shapes were not higher than those with the other marking symbols. Participants in Study $2(N=279)$ received the same graphs, yet without labels and indeed rated the graphs with triangle point markers as more threatening. Testing whether local rather than global spatial attention would lead to an impact of marker shape in climate graphs, Study $3(N=307)$ documented that a task demanding to process a specific data-point on the graph (rather than just the line graph as a whole) did not lead to an effect either. These results suggest that marking symbols can principally affect threat and arousal ratings but not in the context of climate change. Hence, in graphs on climate change, choice of point markers does not have to take potential side-effects on threat and arousal into account. These seem to be restricted to the processing of graphs where form aspects face less competition from the content domain on judgments.
\end{abstract}

Keywords Graph comprehension · Graph characteristics $\cdot$ Marking points $\cdot$ Climate change $\cdot$ Threat $\cdot$ Arousal

\section{Introduction}

Data graphs are one of the most powerful tools to communicate climate change information efficiently. They play an important role when researchers communicate with each other or when results of high societal relevance are communicated in media and scientific textbooks, (see Glazer, 2011, for a review). Many data graph formats had already been developed when industrial production and public management flourished at the end of the nineteenth century (see Friendly, 2008, for a review). Nowadays, in the age of information and technology, the ability to read, understand and critically confront visual data has become even more crucial (Glazer, 2011). Research and communication of climate change is one domain where optimizing graph design

Mariam Katsarava

mariam.katsarava@fernuni-hagen.de

1 Department of Psychology, FernUniversität in Hagen, Universitätsstraße 33, 58097 Hagen, Germany based on the empirical research of graph processing is of high importance (cf. Harold et al., 2016). Empirical work has identified threats to graph comprehension stemming from mappings between content and graph elements that do not match expectations (Fischer et al., 2020) as well as from biases involving emotional and motivational influences on attention (Lewandowsky et al., 2016; Luo \& Zhao, 2019).

Data graphs (and especially line graphs) seem omnipresent in climate research and communication of research on climate change. For instance, in the fifth assessment report of the Intergovernmental Panel on Climate Change (IPCC, 2014), 184 pages of the IPCC5 main chapters (page 111 to page 1246; IPPC, 2014) contain data graphs, 68 of them are line graphs whereas tables displaying quantitative data are comparatively rare (only 24 pages contain such tables). Given that research has shown that graphs aid evaluation of trend (Meyer et al., 1999; Vessey, 1991), it seems justified that graphs are frequently used in research and communication on climate change (Nocke et al., 2008). However, interpretation of graphs can be biased. For instance, 
different parties involved in the public debate on scientific evidence for anthropogenic climate change refer to the same line graphs yet with a different interpretation as to whether there is a clear trend in climate change results (Lewandowsky et al., 2016).

Many studies have tried to identify the most appropriate forms of graphs. For instance, visual features like color, size, animation, use of legend and prior knowledge about the graph content (Brockmann, 1991; Glazer, 2011; Shah \& Hoeffner, 2002) need to be taken into account when constructing data graphs. Optimally, the selection of the graph design should correspond to the data characteristics and the purpose of its presentation. While the above evidence suggests that graphs should be used when representing data on climate change, interpretation of graphs can be biased. One source of bias could stem from design choices. Researchers face a considerable number of options with respect to design choices when translating numerical information into data graphs (Franzblau \& Chung, 2012; Frees \& Miller, 1998; Gelman et al., 2002) which may affect their interpretation. For instance, Fischer et al. (2020) documented that non-intuitive design in an IPPC 2014 graph led to a situation where decision-makers from governmental and non-governmental institutions were at the same time sure and wrong about the interpretation to be drawn. In the counter-intuitive graph, a world map showing the urban population increase until 2050 was combined with boxplots indicating the mid-twenty-first century prediction of the frequency of extreme daily temperature for each region. Yet, higher numerical values (and higher positons on the y-axis for the estimates indicated by boxes) indicated lower levels of climate change impact. Participants misinterpreted the graph by employing their expected mapping rather than the one indicated beneath the figure.

For cases in which it is not possible to identify graph users expectations concerning design (cf. Harold et al., 2016), gestalt principles can help to guide attention to unconventionally useddesign features. For instance, Ali and Peebles (2013) have suggested a technique employing point markers to guide attention to labels on the $\mathrm{x}$-axis or other information that needs to be considered to correct misguiding expectations about the graph layout. In order to avoid the documented problem of ignoring the $\mathrm{x}$-axis in $2 \times 2$ mean value interaction plots, the same colored point markers were displayed on the data points and on the otherwise often ignored axis employing grouping by proximity (Ali \& Peebles, 2013; Kubovy \& van den Berg, 2008).

Some studies suggest that comprehension of data graphs is partially determined by affective responses to the material (cf. Lewandowsky et al., 2016). Apart from content, different visual features such as shape, format and context of the image, can evoke such responses. For instance, various features of images, like roundness, angularity, simplicity, and complexity can predict affective responses to the image
(Lu et al., 2012). Moreover, affective responses can also be influenced by abstract and geometrical shapes, since curved shapes are less arousing than edgy shapes (Pacheco et al., 2015). Bar and Neta (2006) reported in their experiment that pictures of neutral objects with sharp edges were significantly less liked than the same objects with curved contour (e.g., guitar with a curved contour vs. a guitar with sharp edges). Likewise, angular geometric shapes were associated with unpleasant valence (Larson et al., 2012). The importance of shapes was emphasized in a study on car interior design as well-consumers preferred curved forms rather than straight or sharp contours (Leder \& Carbon, 2005).

Preference for specific shapes (e.g., round or circular forms) and the association of some forms (e.g., triangular or pointy shapes) with fear can be explained by theories based on evolutionary psychology (Souchet \& Aubret, 2016). Instant detection of biologically relevant stimuli represents a selective advantage. For instance, some organisms display aposematic signals (i.e., biological warning signals like coloration, sounds, odors, etc.) as a self-defense mechanism. According to Souchet and Aubret (2016), fear of snakes is, in fact, fear of the aposematic signals the snakes are carrying (e.g., dorsal zig-zag patterns, triangular shape of their head, etc.). In order to experimentally tease apart the fear of snakes from aversion of the aposematic signals, children rated pictures of snakes, dogs, and smileys with or without aposematic signals. The animals with aposematic signals (e.g., snake with triangular head) were more frequently rated as "mean" compared to the same pictures without the aposematic signals (e.g., snake with an oval head shape). The authors concluded that humans are more likely to have an innate preparedness to associate aposematic signals with fear, rather than the innate fear of snakes per se. Primates' aversion to potentially harmful aposematic, triangular shapes like teeth, claws, or spikes seems to be exploited by snakes to defend themselves from predators (Souchet \& Aubret, 2016).

Aposematic signals have been associated with processing-advantages on the neural level. Some neuroimaging methods revealed the role of different brain regions and cortical pathways in this process. Generally, visual sensory networks (Dima et al., 2018; You \& Li, 2016), enable the early detection of affective stimuli. Presentation of pictures of objects with sharp edges (Bar \& Neta, 2007) and pointy geometrical shapes (Larson et al., 2009) led to the increased activation of the neural network including the amygdala (Bar \& Neta, 2007), subgenual anterior cingulate cortex, superior temporal gyrus, fusiform gyrus, and extrastriate visual region (Larson et al., 2009). Changes in electrophysiological activation, assessed by Skin Conductance Response and Startle Reflex, turned out to be significantly higher in response to triangles compared to circles (Armbruster et al., 2014). Evidence from a study observing event-related potentials showed that the priming effect of circles and triangles 
influenced the electrophysiological responses to the following stimuli (Wang \& Zhang, 2016).

In sum, according to the above-mentioned studies, triangular shapes draw more attention, are more arousing or unpleasant and evoke a stronger negative bias than round shapes. While past research has documented that edgy shapes can evoke emotional responses even when detached from threatening objects, this potential effect has not been tested in the context of data graphs, for which people frequently select shapes. In many formats of data graphs (i.e., scatterplots, line graphs with point markers), there is a choice between different markers for data points (i.e., circle, rhombus, triangle, etc.). The work on aposematic signals suggests that appropriate point markers should be selected carefully as the emotional reaction to the graph and the evaluation of the graph content may be influenced by the shape of the point markers. Yet, it is not clear whether the design choice of point markers in data graphs can have substantial side effects on the affective reaction to the graph and the evaluation of the graph content. In order to fill this gap, we examined the influence of geometrical figures as point markers on subjective ratings (threat and arousal) of line graph content.

\section{Study 1}

In Study 1, we used data graphs about climate change because these graphs are prevalent in the media and they can elicit considerable emotional reactions (cf. Lewandowsky et al., 2016). The current study tested whether the choice of data point markers matters when using data graphs to convey climate change data to non-experts. We expected higher threat and arousal ratings for graphs with triangles as point markers, than for any other symbol.

\section{Methods}

\section{Participants}

We recruited 335 participants in total, but we had to exclude 21 due to low proficiency in the German language, familiarity with the study object, non-continuous, and/or unserious participation. The remaining participants $(N=314 ; 68 \%$ female; $M_{\text {age }}=33.38, S D=13.56$, range $14-79$ years) were either native German speakers (92\%) or fluent in German $(8 \%)$. Students received partial course credit for their participation. The study was approved by the ethics committee of FernUniversität in Hagen.

\section{Material and Procedure}

Participants were instructed that they would receive data graphs containing veridical climate change data and would be asked to make certain judgments. They gave informed consent before participating in the study and confirmed that the collected data may be used for research purposes again at the end of the survey. Participants answered demographic questions about their age, gender, and knowledge of the German language, before beginning to rate the data graphs. As visual material, we used five different graphs about climate change (glacier mass balance, ${ }^{1}$ global annual ocean temperature, ${ }^{2}$ global annual land surface temperature, ${ }^{3} \mathrm{CO}_{2}$ emissions from fossil fuel energy sources, ${ }^{4}$ and sea level change $^{5}$ ) and five different symbols (triangle, circle, square, rhombus, and asterisk). The line graphs were created in Microsoft Excel. We outlined the data points on the line using one specific symbol. All graphs had the same dimensions and the same layout. The $\mathrm{X}$-axis depicted the years (from 2011 to 2018) and the Y-axis depicted the units of the climate indicator (see Fig. 1). Graphs were presented to the participants one at a time and each graph was followed by two ratings: Subjective threat ("How threatening do you consider the scientific evidence in the graph?") was rated on a scale from 1 (not threatening) to 5 (very threatening). On the second scale, participants rated their subjective arousal ("How arousing do you consider the scientific evidence in the graph?"; 1 - calm to 5 - arousing). After completing the first graph, they had to click "next" to get another graph with the same two subjective ratings. Time to complete the task was not restricted.

\section{Design}

We used a within-subject design with symbol as a factor with five categories (triangles, circles, squares, rhombi, or asterisks). The dependent variables were two subjective ratings (threat and arousal). We controlled for the effect of graph content by pairing each content with each of the five symbols using Latin Square (see Table 1). Each participant responded to five graphs. Among these five graphs, each marker symbol and each content was used exactly once. With this procedure, we ensured that participants were provided with a short and meaningful rating task while we could unobtrusively vary the point markers.

\footnotetext{
1 Glacier mass balance (https://wgms.ch/latest-glacier-mass-balancedata/).

2 Global annual ocean temperature (https://www.ncdc.noaa.gov/sotc/ global/201813).

3 Global annual land surface temperature (https://www.ncdc.noaa. gov/sotc/global/201813).

$4 \mathrm{CO} 2$ emissions from fossil fuel energy sources (https://www.wri. org/blog/2018/12/new-global-co2-emissions- numbers-are-they-renot-good)

55 Sea level change (https://climate.nasa.gov/vital-signs/sea-level/
} 


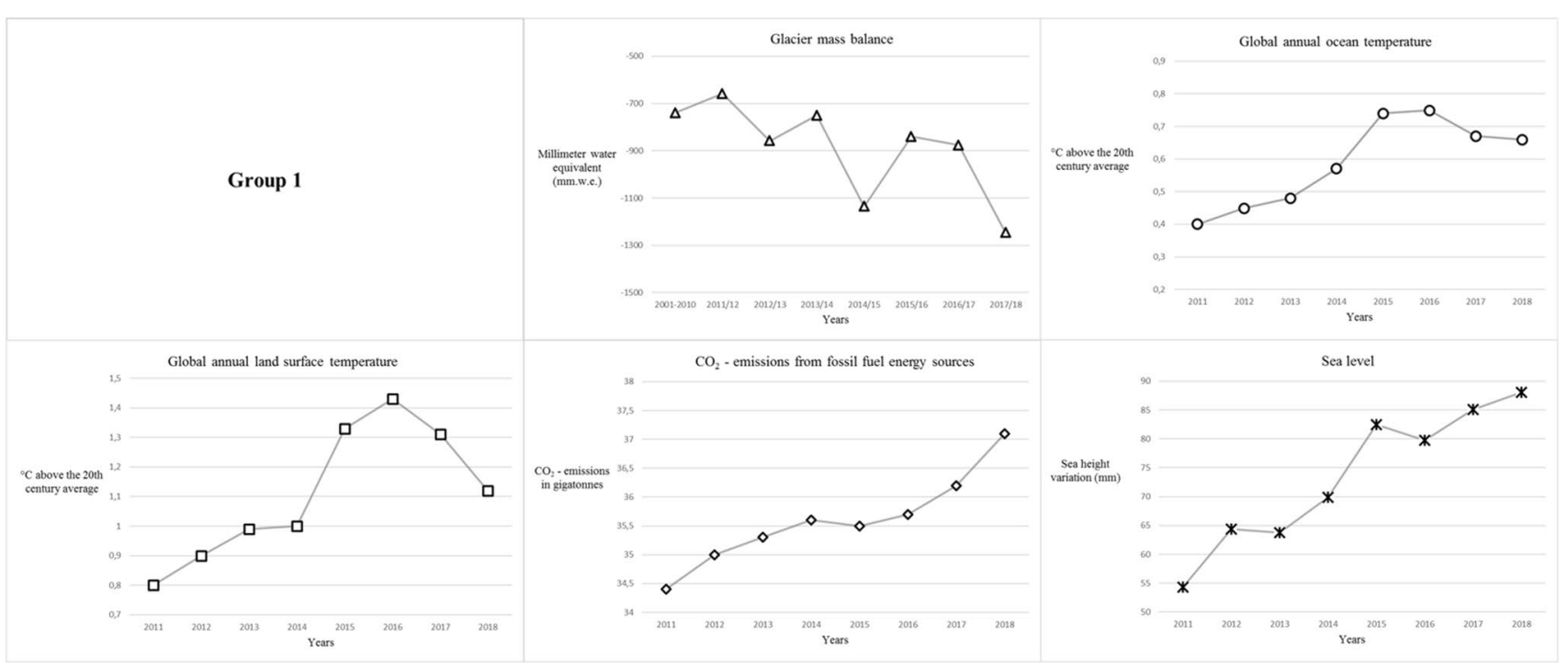

Fig. 1 Presented graph x symbol combinations in the first group

Table 1 Latin Square for balanced graph x symbol combinations across the groups
Glacier mass balance Ocean temperature Land surface $\mathrm{CO}_{2}$ emissions Sea level change temperature

\begin{tabular}{llllll}
\hline Group 1 & Triangle & Circle & Square & Rhombus & Asterisk \\
Group 2 & Asterisk & Triangle & Circle & Square & Rhombus \\
Group 3 & Rhombus & Asterisk & Triangle & Circle & Square \\
Group 4 & Square & Rhombus & Asterisk & Triangle & Circle \\
Group 5 & Circle & Square & Rhombus & Asterisk & Triangle \\
\hline
\end{tabular}

At the beginning of the experiment, participants were randomly assigned to one of the five groups presented in Table 1. For example, participants in the first group received the graph about glacier mass balance with triangles, the graph about ocean temperature with circles, the land surface temperature graph with squares, the $\mathrm{CO}_{2}$ emission graph with rhombi, and the sea level graph with asterisks (see Fig. 1).

To ensure that differences in subjective ratings were primarily influenced by the symbols and not by the sequence of graph presentation, we randomized graph sequence within each group. All graph x symbol combinations and other studyrelated materials are available in the Online Supplementary Material.

\section{Results}

\section{Influence of Geometrical Symbols on the Ratings of the Climate Data}

To investigate the effects of symbols on threat and arousal, repeated measures ANOVAs with symbol as a predictor with five categories (triangles, circles, squares, rhombi, or asterisks) and subjective ratings (threat and arousal) were conducted. Means, standard deviations, and pairwise comparisons are shown in Table 2.

Threat Ratings Means did not differ significantly between the symbols $F(4,1252)=1.03, p=0.392, \eta_{\mathrm{p}}{ }^{2}=0.003$. The rating for triangles did not differ from the one for circles nor from any other point marker (see Table 2). Bayesian repeated measures ANOVA (null/alternative) revealed a value of 333.02:1 in favor of the null hypothesis (with an inverse of $1 / 333.02=0.003$ ). In other words, the data fits the null hypothesis 333 times better, compared to the alternative hypothesis (Faulkenberry et al., 2020).

Arousal Ratings Mean ratings significantly differed between the symbols, $F(4,1252)=2.41, p=0.047, \eta_{\mathrm{p}}{ }^{2}=0.008$. However, the triangles did not diverge from other symbols (see Table 2). The significant symbol main effect was mainly fostered by arousal ratings of rhombi, which were significantly higher compared to the triangles (see Table 2). Bayesian 
Table 2 Pairwise comparisons of threat and arousal ratings for the symbols
Table 3 Influence of the graph content on threat and arousal ratings. Means, standard deviations and p-values of pairwise comparisons

\begin{tabular}{|c|c|c|c|c|c|c|c|c|}
\hline \multirow[b]{2}{*}{ Threat ratings } & \multicolumn{2}{|c|}{ Triangles } & \multicolumn{6}{|c|}{ Other symbols } \\
\hline & $M$ & $S D$ & $M$ & $S D$ & $d f$ & $t$ & $p$ & Cohens $d$ \\
\hline Triangle VS Circle & 3.83 & 1.12 & 3.87 & 1.11 & 313 & -0.60 & 0.546 & -0.03 \\
\hline Triangle VS Square & & & 3.80 & 1.07 & 313 & 0.42 & 0.672 & 0.02 \\
\hline Triangle VS Rhombus & & & 3.90 & 1.05 & 313 & -1.11 & 0.266 & -0.06 \\
\hline Triangle VS Asterisk & & & 3.79 & 1.13 & 313 & 0.60 & 0.552 & 0.03 \\
\hline \multicolumn{9}{|l|}{ Arousal ratings } \\
\hline Triangle VS Circle & 3.86 & 1.13 & 3.90 & 1.10 & 313 & -0.79 & 0.433 & -0.04 \\
\hline Triangle VS Square & & & 3.87 & 1.08 & 313 & -0.19 & 0.851 & -0.01 \\
\hline Triangle VS Rhombus & & & 4.03 & 1.03 & 313 & -2.66 & 0.008 & -0.15 \\
\hline Triangle VS Asterisk & & & 3.87 & 1.12 & 313 & -0.25 & 0.803 & -0.01 \\
\hline
\end{tabular}

\begin{tabular}{|c|c|c|c|c|c|c|c|}
\hline Graphs & $M$ & $S D$ & 1 & 2 & 3 & 4 & 5 \\
\hline \multicolumn{8}{|l|}{ Threat ratings } \\
\hline 1. Glacier mass balance & 4.05 & 1.04 & - & & & & \\
\hline 2. Ocean temperature & 3.65 & 1.03 & $<0.001$ & - & & & \\
\hline 3. Land temperature & 3.27 & 1.08 & $<0.001$ & $<0.001$ & - & & \\
\hline 4. $\mathrm{CO} 2$ emissions & 4.12 & 1.07 & 0.822 & $<0.001$ & $<0.001$ & - & \\
\hline 5. Sea level change & 4.09 & 1.01 & 1.00 & $<0.001$ & $<0.001$ & 1.00 & - \\
\hline \multicolumn{8}{|l|}{ Arousal ratings } \\
\hline 1. Glacier mass balance & 4.12 & 1.03 & - & & & & \\
\hline 2. Ocean temperature & 3.69 & 1.08 & $<0.001$ & - & & & \\
\hline 3. Land temperature & 3.38 & 1.10 & $<0.001$ & $<0.001$ & - & & \\
\hline 4. $\mathrm{CO} 2$ emissions & 4.18 & 1.02 & 0.967 & $<0.001$ & $<0.001$ & - & \\
\hline 5. Sea level change & 4.16 & 0.99 & 1.00 & $<0.001$ & $<0.001$ & 1.00 & - \\
\hline
\end{tabular}

repeated measures ANOVA (null/alternative) suggested that the data fit to the null hypothesis was 24.2:1 (with an inverse of $1 / 24.2=0.04)$. Thus, the data correspond to the null hypothesis 24 times better than to the alternative hypothesis (Faulkenberry et al., 2020).

In sum, we did not find evidence in favor of our hypotheses that triangular symbols increase threat and arousal. Instead, the Bayesian analysis showed that the data provided strong evidence in favor of the null hypothesis.

\section{Influence of the Graph Content on the Ratings of the Climate}

Data In order to rule out that the lack of impact due to point markers was caused by the fact that the data graphs were not examined to a sufficient extent, we analyzed whether there were differences in ratings among the different data graphs based on content rather than type of point marker. This was indeed the case. The repeated measures ANOVA (with Greenhouse-Geisser correction) showed a significant main effect for content on threat, $F(3.9,1213.7)=79.80, p<0.001$, $\eta_{\mathrm{p}}{ }^{2}=0.203$, and arousal ratings, $F(3.8,1183.2)=75.29$, $p<0.001, \eta_{\mathrm{p}}{ }^{2}=0.194$. Pairwise comparisons of graphs on
$\mathrm{CO}_{2}$ emissions from fossil energy sources, variation in sea level, and global average glacier mass balance showed no significant differences (all $p s \geq 0.822$ ) with regards to threat and arousal ratings (see Table 3). However, they were rated as more threatening and arousing than the graphs on global annual ocean temperature and global annual land temperature (all $p s<0.001$ ). In addition, the graph on global annual ocean temperature was even more threatening and arousing than the graph about global annual land temperature (both ps $<0.001)$.

We also analyzed the effect due to the order of graph presentation. As already mentioned above, we randomized the sequence of the graph presentation. This method was used to exclude the effect of order on the subjective ratings. We could not find significant main effects of graph position in the string for threat or for arousal ratings (all $p s>0.08)$. Thus, there was no accumulation of threat or arousal across the graphs that the participants viewed and rated in succession.

Given the age-heterogeneous sample, we explored whether threat or arousal ratings would substantially 
correlate with age. This was not the case. For threat, the range of Spearman rank order correlations with age was -0.115 to 0.032 . For arousal, the range was -0.142 to -0.026 .

\section{Discussion}

The results of Study 1 show that the triangles as point markers of graphs did not influence the subjective threat or arousal ratings of the data concerning climate change. Bayesian analysis showed strong evidence against such an effect, countering the perspective that the data might not be sufficiently powered to detect this. Furthermore, while form did not influence the ratings, there were significant differences depending on the content of the graphs. This rules out the possibility that participants might have failed to pay proper attention to the graphs. Rather, the results show that the point markers did not influence the ratings even though participants processed the content of the graphs. For instance, graphs about $\mathrm{CO}_{2}$ emissions, changes in the sea level, and the glacier mass balance index were perceived as more threatening and arousing than the graphs about the ocean and land temperature. This effect of graph content on threat and arousal provides evidence for participants' discriminative assessments of the graphs. One reason for these different ratings could be the pattern of the curves on the graphs. Trend lines on the graphs about $\mathrm{CO}_{2}$ emissions and changes in the sea level show a gradual increase or decrease, especially in recent years and therefore, the participants could have attributed negative prognostic value to these trends. Such extrapolation of the data trend can affect participants' expectations regarding future perspectives and subsequently impact their threat and arousal ratings (Glazer, 2011; Larson et al., 2012; Wainer, 1992).

Furthermore, the content of the graphs - climate change in general, in addition to the specific aspect transported in the each graph - should have affected the ratings as well. Many studies have documented that prior knowledge, beliefs, cognitive model, and the goals of information search can affect graph comprehension (Chinn \& Brewer, 2001; Schwartz et al., 2007; Winn, 1993). The fact that both ratings (threat and arousal) were high for all five graphs (means were above the midpoint of the scale) indicates that the majority of the participants were concerned about the presented data. This likely made the task of evaluating the data graphs meaningful to them. On the one hand, one could argue that processing graphs in a meaningful domain might increase the likelihood that potential effects of the shapes of the point markers influence the ratings: Participants might search for cues to differentiate the ratings among the different graphs all dealing with an important issue. As ratings were not at the extreme of the scale, there should have been some room for an impact due to the point marker combined with the specific content to influence the ratings. Our results suggest that there is no effect of form when people process data graphs on an issue they highly care about.

On the other hand, one could argue that providing strong statistical evidence against an effect of point markers is not enough. According to the results of Study 1, we cannot conclude whether our findings refer to processing of data graphs in a domain the participants care about (such as climate change) or whether they are relevant for line graphs with point markers in general - no matter whether or not participants care strongly about the content of the graphs. Thus, it is important to test whether the form of point markers influences threat and arousal ratings when the content of the graphs is less involving for the participants.

\section{Study 2}

In order to rule out the possibility that participants' attitudes toward climate change might affect their judgment of graphs' visual characteristics, we conducted Study 2 free of climate change content. We examined threat and arousal ratings, offering participants only the visual characteristics of the graphs. Contrary to Study 1, participants received the unlabeled graphs, so they were not made aware of the domain of the graphs. We expected higher threat and arousal ratings for graphs depicting the triangles as point markers, compared to the graphs with other symbols (see preregistration at osf.io/jqtvg).

\section{Methods}

\section{Participants}

By the scheduled end of data collection we had collected data from 321 participants ( 340 had been planned) and had to exclude 42 due to low proficiency in the German language, participation in Study 1, familiarity with the study object, unserious and/or non-continuous participation. The remaining participants $\left(N=279 ; M_{\text {age }}=30.69, S D=10.24\right.$, range $18-83$ years; $76.7 \%$ female; $22.9 \%$ male; $0.4 \%$ other) were native German speakers $(90 \%)$ or fluent in German $(10 \%)$.

\section{Material, Procedure and Design}

Participants were presented with the same graphs as in Study 1 , yet there were no labels, and the instructions did not mention climate change. In order to provide participants with a task that was also meaningful from their perspective, we informed them that graphs could be used in various domains with, in some cases, threatening or arousing content and 
Table 4 Pairwise comparisons of threat and arousal ratings for the symbols

\begin{tabular}{|c|c|c|c|c|c|c|c|c|}
\hline \multirow[b]{2}{*}{ Threat ratings } & \multicolumn{2}{|c|}{ Triangles } & \multicolumn{6}{|c|}{ Other symbols } \\
\hline & $M$ & $S D$ & $M$ & $S D$ & $d f$ & $t$ & $p^{a}$ & Cohens $d$ \\
\hline Triangle VS Circle & 2.18 & 1.08 & 1.95 & 1.09 & 278 & 3.04 & 0.024 & 0.18 \\
\hline Triangle VS Square & & & 1.97 & 1.04 & 278 & 2.75 & 0.060 & 0.17 \\
\hline Triangle VS Rhombus & & & 2.01 & 1.09 & 278 & 2.17 & 0.301 & 0.13 \\
\hline Triangle VS Asterisk & & & 2.29 & 1.19 & 278 & -1.59 & 1.00 & -0.10 \\
\hline \multicolumn{9}{|l|}{ Arousal ratings } \\
\hline Triangle VS Circle & 2.22 & 1.12 & 2.15 & 1.18 & 278 & 0.84 & 1.00 & 0.05 \\
\hline Triangle VS Square & & & 2.08 & 1.07 & 278 & 1.81 & 0.701 & 0.11 \\
\hline Triangle VS Rhombus & & & 2.13 & 1.13 & 278 & 1.16 & 1.00 & 0.07 \\
\hline Triangle VS Asterisk & & & 2.38 & 1.21 & 278 & -2.05 & 0.411 & -0.12 \\
\hline
\end{tabular}

${ }^{a} p$-values are Bonferroni corrected that threat and arousal might be amplified or dampened by choices in how to set up the graph. Each participant was presented with 5 graphs according to the same Latin Square design as in Study 1 (see Table 1). The questions differed slightly from Study 1: (A) "How threatening does the graph appear to you?"-1 (not threatening) to 5 (very threatening). (B) "How arousing does the graph appear to you?"-1 (calm) to 5 (arousing).

\section{Results}

\section{Influence of Geometrical Symbols on the Ratings of the Climate Data}

Threat Ratings A repeated-measures ANOVA revealed a significant influence of the symbol on threat assessment, $F(4,1112)=7.97, p<0.001, \eta_{\mathrm{p}}{ }^{2}=0.028$. See Table 4 for means, standard deviations, and pairwise comparisons. According to the post-hoc analysis (with Bonferroni correction), the threat rating of triangles was significantly higher compared to the rating of circles $(p=0.024)$. None of the other comparisons revealed significant differences (all $p s \geq 0.060$; see Table 4). The data was additionally examined using Bayesian repeated measures ANOVA (alternative/ null), which showed that the data fits the alternative hypothesis 1642.87 times better, compared to the null hypothesis, with an inverse of $1 / 1642.87=0.00060869$ (Faulkenberry et al., 2020).

Arousal Ratings A repeated-measures ANOVA (with Greenhouse-Geisser correction) revealed a significant main effect of symbols on arousal ratings, $F(3.85,1070.26)=4.45$, $p=0.002, \eta_{\mathrm{p}}{ }^{2}=0.016$. The post-hoc analysis (with Bonferroni correction) revealed that there were no significant differences between triangles and other symbols (all $p \mathrm{~s} \geq 0.411$; see Table 4). Instead, significantly higher arousal ratings of asterisks compared to circles, squares, and rhombi
( $p s \leq 0.040)$ led to the significant main effect. Bayesian repeated measures ANOVA (alternative/null) suggested that the data corresponds to the alternative hypothesis 2.27 times better than to the null hypothesis, with an inverse of $1 / 2.27=0.441$ (Faulkenberry et al., 2020).

As the sample was heterogeneous in terms of age, we calculated Spearman rank order correlations between age and the threat and arousal ratings. There were no substantial correlations. For threat, the range of Spearman rank order correlations with age was -0.161 to -0.085 . For the arousal ratings, the range was -0.161 to -0.067 .

In sum, although the threat ratings were significantly higher for triangles compared to circles, the arousal ratings did not reveal the same relationship between independent and dependent variables. Moreover, there was moderate statistical evidence for arousal ratings of asterisks being significantly higher compared to other symbols (except for triangles).

\section{Discussion}

The results of Study 2 provide first evidence that applying triangular symbols on the line graphs can affect participants' subjective ratings, especially the threat ratings. In line with the work on aposematic signals (i.e., Souchet \& Aubret, 2016), threat ratings were significantly higher for data graphs with triangles compared to data graphs with circles. Interestingly, asterisks had significantly higher arousal ratings than circles, squares, and rhombi. This shows that participants differentiated between the two ratings. However, further work is needed to reveal the processes leading to ratings in unlabeled data graphs. Taken together, the effects documented in Study 2 suffice to emphasize the possible impact of point markers in data graphs on threat and arousal ratings. This supports the view that point markers did not affect threat and arousal ratings in Study 1, because people cared about the topic of climate change. 


\section{Study 3}

While Study 1 provided evidence that marker shape did not influence threat and arousal judgments of climate change data, one important aspect remains to be targeted in Study 3. It has long been acknowledged that data graphs can suit different tasks and processing can be strongly task dependent (cf. Lee et al., 2006; Sarikaya \& Gleicher, 2018). One aspect related to the distribution of spatial attention on the data graph seems particularly relevant to test. Potentially, in Study 1 participants assessed the shape of the line graph at a more global level without paying enough attention to particular data points for the shape of the point markers to influence processing. Given the perspective of Souchet and Aubret (2016) one could have expected automatic influences of marker shape even with a task that does not demand that spatial attention is focused on individual point markers. Yet, the results of Study 1 suggest to test whether point marker shape does at least influence threat and arousal ratings in climate graphs if spatial attention is distributed more locally instead of globally.

There is ample evidence that people can process visual stimuli either predominantly focusing on a more global level or more on a local level (cf. Navon, 1977, 2003). For instance, in the Navon-task, participants process large letters on the screen being composed of small letters. Depending on task or instruction, they can either focus on the small letters (i.e., the local level) or the large letter (i.e., the global level; cf. Lachmann et al., 2014). Furthermore, per default, people seem to be inclined to focus more on the global level (Navon, 1977). Global processing might also have been sufficient to cope with Study 1 task demands. According to the work with the Navon-task, focusing on the more global level (i.e., shape of the line graph as a whole), might have reduced attention to the more local level. This might have limited the amount of attention granted to the shape of the point markers, therefore reducing their potential impact on the threat and arousal rating.

The above reasoning leads to the conclusion that a graphprocessing task demanding local attention would provide stronger potential for an impact of point marker shape on threat- and arousal ratings. Some tasks on line graphs require attention to local data points (signaled by point markers). For instance, people might use the last data point in a line graph for a judgment of the state of the time series. Thus, on the one hand, a task demanding local attention is within the scope of potential uses for climate change data graphs. On the other hand, such a task provides an even stronger test for a potential impact of point marker shape. Testing this perspective could either lead to the result that marker shape has an influence depending on the variant of task processing the climate data graph is subjected to. Or it could lead to the result that point marker shape lacks influence even with a task demanding local attention.

\section{Methods}

\section{Participants}

We collected data from 343 participants. We had to exclude 36 participants due to low proficiency in the German language, participation in other similar studies, familiarity with the study object, unserious and/or non-continuous participation. Approximately $95 \%$ of the remaining participants $\left(N=307 ; M_{\text {age }}=40.95, S D=12.85\right.$, range $19-72$ years; $58.6 \%$ female; $39.7 \%$ male; $1.3 \%$ other) were native German speakers, and about $5 \%$ were fluent in German.

\section{Material, Procedure and Design}

Participants in Study 3 were presented with the identical graphs and identical instruction as in Study 1 . The only difference was the formulation of ratings. Contrary to Study 1 , where participants had to rate how threatening and arousing the scientific evidence in the whole graph was, in Study 3, participants had to make the threat rating based on the situation in the last year depicted on the graph ("Please look at the last data point in the chart, i.e., the glacier mass balance 2017/2018. How threatening do you consider the situation in 2017/2018?" - from 1 [not threatening] to 5 [very threatening]). On the second scale of the same graph, participants had to rate their arousal ("How arousing do you consider the situation in 2017/2018?" - from 1 [not arousing] to 5 [very arousing]).

\section{Results}

\section{Influence of Geometrical Symbols on the Ratings of the Climate Data}

Repeated measures ANOVAs were conducted to examine the effects of symbols (triangles, circles, squares, rhombi, and asterisks) on the threat and arousal ratings concerning the last depicted year. Means, standard deviations, and pairwise comparisons are summarized in Table 5.

Threat Ratings Repeated measures ANOVA (with Greenhouse-Geisser correction) did not reveal significant differences between the mean threat ratings of the symbols $(F<1)$. Pairwise comparisons showed that the threat ratings of triangles and circles, as well as of any other symbols, did not differ significantly (see Table 5). According to the Bayesian repeated measures ANOVA (null/alternative), data fits the null hypothesis much better compared 
Table 5 Pairwise comparisons of threat and arousal ratings for the symbols
Table 6 Influence of the graph content on threat and arousal ratings. Means, standard deviations and $\mathrm{p}^{\mathrm{a}}$-values of pairwise comparisons

\begin{tabular}{|c|c|c|c|c|c|c|c|c|}
\hline \multirow[b]{2}{*}{ Threat ratings } & \multicolumn{2}{|c|}{ Triangles } & \multicolumn{6}{|c|}{ Other symbols } \\
\hline & $M$ & $S D$ & $M$ & $S D$ & $d f$ & $t$ & $p^{a}$ & Cohens $d$ \\
\hline Triangle VS Circle & 3.89 & 1.06 & 3.92 & 1.06 & 306 & -0.53 & 0.599 & -0.03 \\
\hline Triangle VS Square & & & 3.90 & 1.09 & 306 & -0.20 & 0.843 & -0.01 \\
\hline Triangle VS Rhombus & & & 3.88 & 1.07 & 306 & 0.18 & 0.856 & 0.01 \\
\hline Triangle VS Asterisk & & & 3.86 & 1.06 & 306 & 0.42 & 0.673 & 0.02 \\
\hline \multicolumn{9}{|l|}{ Arousal ratings } \\
\hline Triangle VS Circle & 4.02 & 1.06 & 4.03 & 1.06 & 306 & -0.16 & 0.872 & -0.01 \\
\hline Triangle VS Square & & & 3.93 & 1.12 & 306 & 1.47 & 0.143 & 0.08 \\
\hline Triangle VS Rhombus & & & 3.96 & 1.08 & 306 & 0.85 & 0.395 & 0.05 \\
\hline Triangle VS Asterisk & & & 3.99 & 1.12 & 306 & 0.52 & 0.607 & 0.03 \\
\hline
\end{tabular}

\begin{tabular}{llllllll}
\hline Graphs & $M$ & $S D$ & 1 & 2 & 3 & 4 & 5 \\
\hline Threat ratings & & & & & & & \\
1. Glacier mass balance & 4.18 & 1.00 & - & & & \\
2. Ocean temperature & 3.63 & 0.99 & $<0.001$ & - & & \\
3. Land temperature & 3.30 & 0.99 & $<0.001$ & $<0.001$ & - & - \\
4. CO2 emissions & 4.24 & 1.00 & 0.289 & $<0.001$ & $<0.001$ & - \\
5. Sea level change & 4.09 & 1.04 & 0.223 & $<0.001$ & $<0.001$ & 0.024 \\
Arousal ratings & & & & & & \\
1. Glacier mass balance & 4.26 & 1.02 & - & & & \\
2. Ocean temperature & 3.77 & 1.05 & $<0.001$ & - & & \\
3. Land temperature & 3.43 & 1.06 & $<0.001$ & $<0.001$ & - & \\
4. CO2 emissions & 4.33 & 0.98 & 0.181 & $<0.001$ & $<0.001$ & - \\
5. Sea level change & 4.15 & 1.08 & 0.126 & $<0.001$ & $<0.001$ & 0.004 & - \\
\hline
\end{tabular}

to the alternative hypothesis (1382.15; with an inverse of $1 / 1382.15=0.00072351$; Faulkenberry et al., 2020).

Arousal Ratings Repeated measures ANOVA (with Greenhouse-Geisser correction) did not show significant symbol main effect on arousal ratings $(F<1)$. Thus, means did not differ significantly between the symbols (see Table 5). Bayesian repeated measures ANOVA (null/alternative) revealed strong evidence in favour of the null hypothesis (406.44; with an inverse of $1 / 406.44=0.00246039$; Faulkenberry et al., 2020).

Influence of the Graph Content on the Ratings of the Climate Data Similarly to Study 1, we tested the differences in subjective ratings made for different graph contents (Glacier mass balance, Ocean temperature, Land surface temperature, $\mathrm{CO} 2$ emissions, and Sea level change). The results suggest that graphs were indeed examined and rated differently based on their content. The repeated measures ANOVA (with Greenhouse-Geisser correction) revealed a significant main effect for content on threat, $F(3.8$, $1153.5)=108.53, p<0.001, \eta_{\mathrm{p}}{ }^{2}=0.262$, and arousal ratings,
$F(3.7,1135)=92.66, p<0.001, \eta_{\mathrm{p}}{ }^{2}=0.232$. Pairwise comparisons showed that the graph on global average glacier mass balance did not differ from the graph on $\mathrm{CO}_{2}$ emissions from fossil energy sources or graph on variation in sea level significantly (all $p s \geq$. 126) regarding the threat or arousal ratings (see Tables 6). However, these graphs were rated as more threatening and arousing than the graphs on global annual ocean temperature and global annual land temperature (all $p \mathrm{~s}<0.001$ ). Threat and arousal ratings of graph on $\mathrm{CO}_{2}$ emissions were significantly higher compared to the ratings of graph on variation in sea level (all $p$ s $\leq 0.024$; see Table 6).

\section{Discussion}

Replicating Study 1, we again found that shape of point marker did not influence threat and arousal ratings when processing line graphs on climate change. While the effect of graph content again confirmed that participants had indeed processed the graphs, the absence of a shape effect in Study 3 substantially extends the finding of Study 1: Shape lacked an influence even though this time the task demanded a more 
local processing mode. In Study 3, participants more likely focused on particular point markers due to the instruction to base their judgment on the last value of the time course. While a more global processing mode might have reduced the attention allocated to the markers, this potential explanation for the lack of impact of the marker shapes was ruled out in Study 3. Apparently, whenever participants are provided with information on the content of the data graphs (Study 1 and Study 3), graph shapes have no weight. As this seems to count for different tasks done with line graphs, marker shapes might be chosen to optimize discriminability of data in the task at hand rather than with an eye on avoiding potentially threat-laden shapes as suggested by the perspective of Souchet and Aubret (2016).

\section{General Discussion}

The present work aimed at examining the influence of triangular shapes on threat perception. While work on aposematic signals suggests that triangular shapes might be associated with threat and arousal (Souchet \& Aubret, 2016), triangular point markers did not add to the threat and arousal reported with respect to climate change graphs in Study 1. However, graph symbols affected threat and arousal ratings in Study 2 , when graphs were presented without climate labels. Study 3 showed that marker shape did not influence ratings of climate change graphs even when the task demanded attending single data points. Thus, the lack of influence of maker shape was robust to variations in the task performed with the climate change graphs and even found when more local distribution of spatial attention was demanded.

When content was absent (Study 2), ratings were lower than in Study 1 and 3 and were influenced by the form of the point marker. Taken together, this suggests that the impact of aposematic signals might be restricted by the context. Relevant for practical considerations, the processing of graphs displaying content that is important and meaningful to people does not seem to be influenced by the form of the point markers. The study thus adds to the empirical work on optimizing data graph design and usage in climate research and research communication (e.g., Fischer et al., 2020; Harold et al., 2016; Lewandowsky et al., 2016; Luo \& Zhao, 2019). For instance, design choices with respect to point markers can focus on optimizing visual discriminability, as this aspect does not have to be weighed against the potential effect of aposematic signaling. The current study suggests that the latter has no influence when people process graphs on climate change.

Following up on Study 2, future research should identify additional conditions under which aposematic signals have an impact on data graph processing. Strong involvement in the content might override (rather than aggravate) influences of form. It is conceivable that form influences are restricted to tasks that ask about aspects of the shape. In Souchet and Aubret (2016) as well as in Study 2 participants had nothing but the graphical characteristics to base their judgment on. Potentially, the influence of aposematic signaling is weak and only plays out when there is no competition by domain knowledge and attitudes. It is conceivable that irrelevant graphical aspects are ignored in favor of relevant aspects - as long as the latter are present in the graph. This would be in line with top-down discarding of irrelevant information (cf. Gaschler et al., 2010, 2015).

Affective priming studies have shown that the affective evaluation of one stimulus can influence the evaluation of another stimulus (Payne et al., 2005; Wong \& Root, 2003). Affect (caused by the first stimulus) seems to be used as information when judging the quality of another stimulus or other aspects of the same stimulus (Schwarz \& Clore, 1983; Winkielman et al., 2007). Hence, affective reactions to specific shapes in data graphs may influence the evaluation of other aspects of the graphs such as the graph content. To follow up on this, future work on the impact of aposematic signaling might use the framework of parallel constraint satisfaction in coherence-based reasoning (cf. Glöckner \& Betsch, 2008; Glöckner et al., 2014). This work uses neural network modelling to capture how and when irrelevant aspects can influence judgments and could in the future be applied to data graph processing. In particular, irrelevant aspects (i.e., an aposematic shape) pointing in the same direction as the relevant aspect (i.e., threatening content) can lead to aggravated judgments (i.e., higher threat rating on a climate graph). This happens because activation is fed back in case of coherence in cycles of recurrent processing in the network. Iterative cycles of activation support and emphasize features that are coherent with other characteristics at the cost of relevant aspects not being supported by an irrelevant aspect pointing in the same direction. Modelling makes specific predictions on how judgments and judgment time should be related. Adding irrelevant but coherent characteristics to a graph should lead to shorter reaction times and stronger judgments (cf. Heck \& Erdfelder, 2017; Jekel et al., 2018). Another interesting finding of both studies was the heightened arousal ratings for rhombi (Study 1) and asterisks (Study 2). Future studies might examine whether higher arousal ratings of these symbols can be explained by their resemblance to aposematic signals.

One limitation of our studies could be the direction of the triangles. We used upward-pointing triangles, as these are most frequent in data graphs. Yet, downward-pointing (V-shaped) triangles may be associated with higher threat ratings (Larson et al., 2009). Armbruster et al. (2014) assessed the physiological markers activated in response to geometric shapes (circles, upward- and downward-pointing 
triangles). The authors reported that the downward-pointing triangles were perceived as less pleasant and more arousing compared to the other geometric figures. Likewise, faces with V-shaped eyebrows were detected quicker than faces with inverted V-shaped eyebrows (Tipples et al., 2002). Thus, future studies in this field should take into account that the data content and the form of triangles (upward or downward-pointed) could affect graph comprehension as well as threat and arousal ratings.

\section{Conclusion}

Line graphs are used to communicate data on time courses in domains such as climate change. We tested the potential implications of work on aposematic signaling, suggesting that triangular shapes evoke automatic negative evaluations and arousal for a simple but frequent design choice when producing line graphs. Such an effect was not found when participants processed data graphs that are meaningful for applied settings (i.e., dealing with data on climate change in the media). The results suggest that the form of markers in climate change graphs can be chosen freely, as they do not influence its evaluation.

Supplementary Information The online version contains supplementary material available at https://doi.org/10.1007/s12144-021-02553-2.

Funding Open Access funding enabled and organized by Projekt DEAL

Data Availability All raw data and the study related material can be found at https://osf.io/6wdge/. Preregistration of Study 2 can be found at osf.io/jqtvg.

Code availability Not applicable.

\section{Declarations}

Ethical Approval All procedures performed in the present study that involved human participants were conducted in accordance with the ethical standards of the institutional and/or national research committee and with the 1964 Helsinki declaration and its later amendments or comparable ethical standards.

Informed Consent Informed consent was obtained from all individual participants included in the study.

Disclosure Statement All authors declare that they have no conflict of interest.

Open Access This article is licensed under a Creative Commons Attribution 4.0 International License, which permits use, sharing, adaptation, distribution and reproduction in any medium or format, as long as you give appropriate credit to the original author(s) and the source, provide a link to the Creative Commons licence, and indicate if changes were made. The images or other third party material in this article are included in the article's Creative Commons licence, unless indicated otherwise in a credit line to the material. If material is not included in the article's Creative Commons licence and your intended use is not permitted by statutory regulation or exceeds the permitted use, you will need to obtain permission directly from the copyright holder. To view a copy of this licence, visit http://creativecommons.org/licenses/by/4.0/.

\section{References}

Ali, N., \& Peebles, D. (2013). The effect of gestalt laws of perceptual organization on the comprehension of three-variable bar and line graphs. Human Factors, 55(1), 183-203. https://doi.org/10.1177/ 0018720812452592

Armbruster, D., Suchert, V., Gärtner, A., \& Strobel, A. (2014). Threatening shapes: The impact of simple geometric configurations on peripheral physiological markers. Physiology and Behavior, 215221. https://doi.org/10.1016/j.physbeh.2014.06.020

Bar, M., \& Neta, M. (2006). Humans prefer curved visual objects. Psychological Science, 17(8), 645-648. https://doi.org/10.1111/j. 1467-9280.2006.01759.x

Bar, M., \& Neta, M. (2007). Visual elements of subjective preference modulate amygdala activation. Neuropsychologia, 45(10), 21912200. https://doi.org/10.1016/j.neuropsychologia.2007.03.008

Brockmann, R. J. (1991). The unbearable distraction of color. IEEE Transactions on Professional Communication, 34(3), 153-159. https://doi.org/10.1109/47.84109

Chinn, C. A., \& Brewer, W. F. (2001). Models of data: A theory of how people evaluate data. Cognition and Instruction, 19(3), 232-293. https://doi.org/10.1207/S1532690XCI1903_3

Dima, D. C., Perry, G., Messaritaki, E., Zhang, J., \& Singh, K. D. (2018). Spatiotemporal dynamics in human visual cortex rapidly encode the emotional content of faces. Human Brain Mapping, 39(10), 3993-4006. https://doi.org/10.1002/hbm.24226

Faulkenberry, T. J., Ly, A., \& Wagenmakers, E. J. (2020). Bayesian inference in numerical cognition: A tutorial using JASP. Journal of Numerical Cognition, 6(2), 231-259. https://doi.org/10.5964/ jnc.v6i2.288

Fischer, H., Van den Broek, K., Ramisch, K., \& Okan, Y. (2020). When IPCC graphs can foster or bias understanding: Evidence among decision-makers from governmental and non-governmental institutions. Environmental Research Letters, 15(11), 114041. https:// doi.org/10.1088/1748-9326/abbc3c

Franzblau, L. E., \& Chung, K. C. (2012). Graphs, tables, and figures in scientific publications: The good, the bad, and how not to be the latter. Journal of Wrist Surgery, 37(3), 591-596. https://doi.org/ 10.1016/j.jhsa.2011.12.041

Frees, E. W., \& Miller, R. B. (1998). Designing effective graphs. North American Actuarial Journal, 2(2), 53-70. https://doi.org/10.1080/ 10920277.1998.10595699

Friendly, M. (2008). The golden age of statistical graphics. Statistical Science, 23(4), 502-535. https://doi.org/10.1214/08-STS268

Gaschler, R., Marewski, J. N., \& Frensch, P. A. (2015). Once and for all - How people change strategy to ignore irrelevant information in visual tasks. Quarterly Journal of Experimental Psychology, 68, 543-567. https://doi.org/10.1080/17470218.2014.961933

Gaschler, R., Mata, J., Störmer, V., Kühnel, A., \& Bilalic, M. (2010). Change detection for new food labels. Food Quality and Preference, 21, 140-147. https://doi.org/10.1016/j.foodqual.2009.08.013

Gelman, A., Pasarica, C., \& Dodhia, R. (2002). Let's practice what we preach: Turning tables into graphs. The American Statistician, 56(2), 121-130. https://doi.org/10.1198/000313002317572790

Glazer, N. (2011). Challenges with graph interpretation: A review of the literature. Studies in Science Education, 47(2), 183-210. https://doi.org/10.1080/03057267.2011.605307 
Glöckner, A., \& Betsch, T. (2008). Multiple-reason decision making based on automatic processing. Journal of Experimental Psychology: Learning, Memory, and Cognition, 34, 1055-1075. https:// doi.org/10.1037/0278-7393.34.5.1055

Glöckner, A., Hilbig, B. E., \& Jekel, M. (2014). What is adaptive about adaptive decision making? A parallel constraint satisfaction account. Cognition, 133, 641-666. https://doi.org/10.1016/j. cognition.2014.08.017

Harold, J., Lorenzoni, I., Shipley, T. F., \& Coventry, K. R. (2016). Cognitive and psychological science insights to improve climate change data visualization. Nature Climate Change, 6(12), 10801089. https://doi.org/10.1038/nclimate 3162

Heck, D. W., \& Erdfelder, E. (2017). Linking process and measurement models of recognition-based decisions. Psychological Review, 124, 442-471. https://doi.org/10.1037/rev0000063

IPCC (2014). Climate Change 2014: Mitigation of Climate Change. Contribution of Working Group III to the Fifth Assessment Report of the Intergovernmental Panel on Climate Change [Edenhofer, O., Pichs-Madruga, R., Sokona, Y., Farahani, E. , Kadner, S., Seyboth, K., Adler, A., Baum, I., Brunner, S., Eickemeier, P., Kriemann, B., Savolainen, J., Schlömer, S., von Stechow, C., Zwickel, T., and Minx, J. C. (eds.)]. Cambridge University Press, Cambridge, United Kingdom and New York, NY, USA.

Jekel, M., Glöckner, A., \& Bröder, A. (2018). A new and unique prediction for cue-search in a parallel-constraint satisfaction network model: The attraction search effect. Psychological Review, 125, 744-768. https://doi.org/10.1037/rev0000107

Kubovy, M., \& Van den Berg, M. (2008). The whole is equal to the sum of its parts: A probabilistic model of grouping by proximity and similarity in regular patterns. Psychological Review, 115(1), 131-154. https://doi.org/10.1037/0033-295X.115.1.131

Lachmann, T., Schmitt, A., Braet, W., \& van Leeuwen, C. (2014). Letters in the forest: Global precedence effect disappears for letters but not for non-letters under reading-like conditions. Frontiers in Psychology, 5, 705. https://doi.org/10.3389/fpsyg.2014.00705

Larson, C. L., Aronoff, J., Sarinopoulos, I. C., \& Zhu, D. C. (2009). Recognizing threat: A simple geometric shape activates neural circuitry for threat detection. Journal of Cognitive Neuroscience, 21(8), 1523-1535. https://doi.org/10.1162/jocn.2009.21111

Larson, C. L., Aronoff, J., \& Steuer, E. L. (2012). Simple geometric shapes are implicitly associated with affective value. Motivation and Emotion, 36, 404-418. https://doi.org/10.1007/ s11031-011-9249-2

Leder, H., \& Carbon, C. C. (2005). Dimensions in appreciation of car interior design. Applied Cognitive Psychology, 19(5), 603-618. https://doi.org/10.1002/acp.1088

Lee, B., Plaisant, C., Parr, C.S., Fekete, J.-D., Henry, N. (2006). Task taxonomy for graph visualization. In BELIV Workshop, 1-56. https://doi.org/10.1145/1168149.1168168

Lewandowsky, S., Ballard, T., Oberauer, K., \& Benestad, R. (2016). A blind expert test of contrarian claims about climate data. Global Environmental Change, 39, 91-97. https://doi.org/10.1016/j.gloen vcha.2016.04.013

Lu, X., Suryanarayan, P., Adams, R. B., Jr, Li, J., Newman, M. G., \& Wang, J. Z. (2012). On shape and the computability of emotions. In Proceedings of the 20th ACM International Conference on Multimedia, 229-238. https://doi.org/10.1145/2393347.2393384

Luo, Y., \& Zhao, J. (2019). Motivated attention in climate change perception and action. Frontiers in Psychology, 10, 1541. https://doi. org/10.3389/fpsyg.2019.01541

Meyer, J., Shamo, M. K., \& Gopher, D. (1999). Information structure and the relative efficacy of tables and graphs. Human Factors, 41(4), 570-587. https://doi.org/10.1518/001872099779656707
Navon, D. (1977). Forest before trees: The precedence of global features in visual perception. Cognitive Psychology, 9(3), 353-383. https://doi.org/10.1016/0010-0285(77)90012-3

Navon, D. (2003). What does a compound letter tell the psychologist's mind? Acta Psychologica, 114(3), 273-309. https://doi.org/10. 1016/j.actpsy.2003.06.002

Nocke, T., Sterzel, T., Böttinger, M., \& Wrobel, M. (2008). Visualization of climate and climate change data: An overview. Digital Earth Summit on Geoinformatics, 226-232.

Pacheco, D., Le Groux, S., \& Verschure, P. F. (2015). Two dimensional shapes for emotional interfaces: Assessing the influence of angles, curvature, symmetry and movement. $A C H I, 224-228$. https://doi. org/10.13140/RG.2.1.4549.6801

Payne, B. K., Cheng, C. M., Govorun, O., \& Stewart, B. D. (2005). An inkblot for attitudes: Affect misattribution as implicit measurement. Journal of Personality and Social Psychology, 89(3), 277-293. https://doi.org/10.1037/0022-3514.89.3.277

Sarikaya, A., \& Gleicher, M. (2018). Scatterplots: Tasks, data, and designs. IEEE Transactions on Visualization \& Computer Graphics, 24(1), 402-412. https://doi.org/10.1109/TVCG.2017.2744184

Schwartz, D. L., Sears, D., \& Chang, J. (2007). Reconsidering prior knowledge. Thinking with data: 33rd Carnegie Symposium on Cognition (319-344). Lawrence Erlbaum Associates Publishers.

Schwarz, N., \& Clore, G. L. (1983). Mood, misattribution, and judgments of well-being: Informative and directive functions of affective states. Journal of Personality and Social Psychology, 45(3), 513-523. https://doi.org/10.1037/0022-3514.45.3.513

Shah, P., \& Hoeffner, J. (2002). Review of graph comprehension research: Implications for instruction. Educational Psychology Review, 14(1), 47-69. https://doi.org/10.1023/A:1013180410169

Souchet, J., \& Aubret, F. (2016). Revisiting the fear of snakes in children: The role of aposematic signalling. Scientific Reports, 6(1), 1-7. https://doi.org/10.1038/srep37619

Tipples, J., Atkinson, A. P., \& Young, A. W. (2002). The eyebrow frown: A salient social signal. Emotion, 2(3), 288-296. https:// doi.org/10.1037/1528-3542.2.3.288

Vessey, I. (1991). Cognitive fit: A theory-based analysis of the graphs versus tables literature. Decision Sciences, 22(2), 219-240. https://doi.org/10.1111/j.15405915.1991.tb00344.x

Wainer, H. (1992). Understanding graphs and tables. Educational Researcher, 21(1), 14-23. https://doi.org/10.3102/0013189X02 $1001014 \% 0 \mathrm{~A}$

Wang, Y., \& Zhang, Q. (2016). Affective priming by simple geometric shapes: Evidence from event-related brain potentials. Frontiers in Psychology, 7, 917. https://doi.org/10.3389/fpsyg.2016.00917

Winkielman, P., Knutson, B., Paulus, M., \& Trujillo, J. L. (2007). Affective influence on judgments and decisions: Moving towards core mechanisms. Review of General Psychology, 11(2), 179-192. https://doi.org/10.1037/1089-2680.11.2.179

Winn, W. (1993). An account of how readers search for information in diagrams. Contemporary Educational Psychology, 18(2), 162185. https://doi.org/10.1006/ceps.1993.1016

Wong, P. S., \& Root, J. C. (2003). Dynamic variations in affective priming. Consciousness and Cognition: An International Journal, 12(2), 147-168. https://doi.org/10.1016/S1053-8100(03)00007-2

You, Y., \& Li, W. (2016). Parallel processing of general and specific threat during early stages of perception. Social Cognitive and Affective Neuroscience, 11(3), 395-404. https://doi.org/10.1093/ scan/nsv123

Publisher's note Springer Nature remains neutral with regard to jurisdictional claims in published maps and institutional affiliations. 